\title{
Determining SUSY particle mixing with polarized hadron beams
}

\author{
Michael Klasen* \\ Laboratoire de Physique Subatomique et de Cosmologie, Université Joseph Fourier I \\ CNRS-IN2P3 / INPG, 53 Avenue des Martyrs, F-38026 Grenoble, France \\ E-mail: klasen@lpsc.in2p3.fr
}

While SUSY particles, if they exist at the TeV-scale, will be discovered at the Tevatron or the LHC, the determination of the SUSY-breaking scenario and its free parameters will require additional information, e.g. from a future International Linear Collider. We point out that such information, in particular on SUSY-particle mixing and the associated soft SUSY-breaking parameters, can also be obtained from measurements at existing or future polarized hadron colliders, since the polarization of initial-state quarks, transmitted through weak gauge bosons or squarks, can be strongly correlated with the helicity and gaugino/higgsino mixing of final-state sleptons, squarks, neutralinos and charginos.

XVIII International Workshop on Deep-Inelastic Scattering and Related Subjects April 19 -23, 2010

Convitto della Calza, Firenze, Italy

${ }^{*}$ Speaker. 


\section{Motivation}

Weak-scale supersymmetry (SUSY) continues to be one of the best-motivated extensions of the Standard Model (SM) of particle physics, and the search for SUSY particles is one of the top priorities at current high-energy hadron colliders. While SUSY particles, if they exist at the TeVscale, will be discovered at the Tevatron or the LHC and their masses and some of their properties will ultimately be measured with an accuracy of about $10 \%$ in the high-energy and high-luminosity phase of the LHC, it is today unanimously accepted that the determination of the SUSY-breaking scenario as well as its free parameters will require additional and more precise measurements, e.g. from a future International Linear Collider [1]. In this article, we point out that additional information, in particular on SUSY-particle mixing and the associated soft SUSY-breaking parameters, may also be obtained from measurements at existing or future polarized hadron colliders such as RHIC or the Tevatron and LHC after polarization upgrades [2].

\section{SUSY particle mixing}

Since the two SUSY partners of a chiral lepton or quark doublet are scalars and carry identical quantum numbers, left- and right-handed sleptons $\tilde{l}_{L, R}$ or squarks $\tilde{q}_{L, R}$ can mix into lighter and heavier mass eigenstates $\tilde{l}_{1,2}$ and $\tilde{q}_{1,2}$. The mixing is proportional to the off-diagonal elements of the sfermion mass matrix

$$
\mathscr{M}^{2}=\left(\begin{array}{cc}
m_{L L}^{2}+m_{f}^{2} & m_{f} m_{L R}^{*} \\
m_{f} m_{L R} & m_{R R}^{2}+m_{f}^{2}
\end{array}\right)
$$

with

$$
\begin{aligned}
& m_{L L}^{2}=\left(T_{f}^{3}-e_{f} \sin ^{2} \theta_{W}\right) m_{Z}^{2} \cos 2 \beta+m_{\tilde{L}}^{2}, \\
& m_{R R}^{2}=e_{f} \sin ^{2} \theta_{W} m_{Z}^{2} \cos 2 \beta+ \begin{cases}m_{\tilde{U}}^{2} & \text { for up - type fermions, } \\
m_{\tilde{D}}^{2} & \text { for down - type sfermions },\end{cases} \\
& m_{L R}=A_{f}-\mu^{*} \begin{cases}\cot \beta & \text { for up - type sfermions } \\
\tan \beta & \text { for down - type sfermions }\end{cases}
\end{aligned}
$$

and thus to the mass of the SM fermion $m_{f}$ and the soft SUSY-breaking term $A_{f}$ of the trilinear Higgs-sfermion-sfermion interaction and the off-diagonal Higgs mass parameter $\mu$ in the MSSM Lagrangian, divided or multiplied by $\tan \beta=s_{\beta} / c_{\beta}=v_{u} / v_{d}$, the ratio of the vacuum expectation values $v_{u, d}$ of the two Higgs doublets. This mass matrix is diagonalized by a unitary matrix

$$
S=\left(\begin{array}{cc}
\cos \theta_{\tilde{f}} \sin \theta_{\tilde{f}} \\
-\sin \theta_{\tilde{f}} \cos \theta_{\tilde{f}}
\end{array}\right) \text { with }\left(\begin{array}{c}
\tilde{f}_{1} \\
\tilde{f}_{2}
\end{array}\right)=S\left(\begin{array}{c}
\tilde{f}_{L} \\
\tilde{f}_{R}
\end{array}\right),
$$

where the mixing angle $\theta_{\tilde{f}} \in[0 ; \pi / 2]$ is related to the SUSY-breaking masses $m_{\tilde{L}, \tilde{U}, \tilde{D}}$ through

$$
\tan 2 \theta_{\tilde{f}}=\frac{2 m_{f} m_{L R}}{m_{L L}^{2}-m_{R R}^{2}}
$$

and the squared mass eigenvalues are given by

$$
m_{1,2}^{2}=m_{f}^{2}+\frac{1}{2}\left(m_{L L}^{2}+m_{R R}^{2} \mp \sqrt{\left(m_{L L}^{2}-m_{R R}^{2}\right)^{2}+4 m_{f}^{2}\left|m_{L R}\right|^{2}}\right) .
$$


If these masses are known, a determination of the sfermion mixing angle will therefore primarily constrain the SUSY and Higgs parameters $A_{f}, \mu$ and $\tan \beta$.

Since the SUSY partners of the neutral and charged electroweak gauge and Higgs bosons are all spin- $1 / 2$ fermions, they can also mix to form four neutral and two charged mass eigenstates $\tilde{\chi}_{i}^{0}$ and $\tilde{\chi}_{i}^{ \pm}$. The neutralino mass matrix

$$
Y=\left(\begin{array}{cccc}
M_{1} & 0 & -m_{Z} s_{W} c_{\beta} & m_{Z} s_{W} s_{\beta} \\
0 & M_{2} & m_{Z} c_{W} c_{\beta} & -m_{Z} c_{W} s_{\beta} \\
-m_{Z} s_{W} c_{\beta} & m_{Z} c_{W} c_{\beta} & 0 & -\mu \\
m_{Z} s_{W} s_{\beta} & -m_{Z} c_{W} s_{\beta} & -\mu & 0
\end{array}\right)
$$

and the chargino mass matrix

$$
X=\left(\begin{array}{cc}
M_{2} & m_{W} \sqrt{2} s_{\beta} \\
m_{W} \sqrt{2} c_{\beta} & \mu
\end{array}\right)
$$

depend not only $\mu$ and $\tan \beta$, but also on $M_{1}$ and $M_{2}$, the SUSY-breaking $B$-ino and $W$-ino mass parameters, while $m_{Z}$ and $m_{W}$ are the SM $Z$ - and $W$-boson masses and $s_{W}\left(c_{W}\right)$ is the sine (cosine) of the electroweak mixing angle $\theta_{W}$. After electroweak gauge-symmetry breaking and diagonalization of the mass matrix $Y$ with a unitary rotation matrix $N$, one obtains the four neutralino mass eigenstates. Their decomposition into gaugino and higgsino components is of major importance, in particular for the determination of the nature of dark matter. The application of projection operators leads to relatively compact analytic expressions for the mass eigenvalues. Since $X$ is not symmetric, it must be diagonalized by two unitary matrices $U$ and $V$. Its squared eigenvalues are

$$
m_{\tilde{\chi}_{1,2}^{ \pm}}^{2}=\frac{1}{2}\left\{\left|M_{2}\right|^{2}+|\mu|^{2}+2 m_{W}^{2} \mp \sqrt{\left(\left|M_{2}\right|^{2}+|\mu|^{2}+2 m_{W}^{2}\right)^{2}-4\left|\mu M_{2}-m_{W}^{2} s_{2 \beta}\right|^{2}}\right\},
$$

and the rotation angle $\theta_{+} \in[0 ; \pi]$ of $V$ is uniquely fixed by the two conditions

$$
\begin{aligned}
\tan 2 \theta_{+} & =\frac{2 \sqrt{2} m_{W}\left(M_{2}^{*} s_{\beta}+\mu c_{\beta}\right) e^{i \phi_{+}}}{\left|M_{2}\right|^{2}-|\mu|^{2}+2 m_{W}^{2} c_{2 \beta}} \text { and } \\
\sin 2 \theta_{+} & =\frac{-2 \sqrt{2} m_{W}\left(M_{2}^{*} s_{\beta}+\mu c_{\beta}\right) e^{i \phi_{+}}}{\sqrt{\left(\left|M_{2}\right|^{2}-|\mu|^{2}+2 m_{W}^{2} c_{2 \beta}\right)^{2}+8 m_{W}^{2}\left[\left(M_{2}^{*} s_{\beta}+\mu c_{\beta}\right) e^{i \phi_{+}}\right]^{2}}} .
\end{aligned}
$$

If the lightest chargino mass $m_{\tilde{\chi}_{1}^{ \pm}}$is known, $|\mu|$ can be determined as a function of $M_{2}$ from Eq. (2.10). The anomalous magnetic moment of the muon and rare $B$-decays both favor $\mu>0$. In addition, one often assumes not only gauge coupling, but also gaugino mass unification at the GUT scale and therefore $M_{1}=5 / 3 \tan ^{2} \theta_{W} M_{2} \simeq 0.5 M_{2}$ at the electroweak scale, leaving $M_{2}$ as the only free parameter determining the gaugino/higgsino decomposition of the neutralinos and charginos.

\section{Correlation with beam polarization}

Our main observation is that the polarization of initial-state quarks, transmitted through $s$ channel exchanges of weak bosons or $t$ - and $u$-channel exchanges of squarks, can be strongly 
correlated with the helicity and gaugino/higgsino mixing of final-state sfermions and neutralinos/charginos. While the partonic double-spin asymmetry for $s$-channel produced sleptons $A_{L L}=-1$ is independent of all SUSY parameters, the single-spin asymmetry $A_{L}=\mathrm{d} \Delta \sigma_{L} / \mathrm{d} \sigma$ with

$$
\mathrm{d} \Delta \sigma_{L}=\frac{4 \pi \alpha^{2}}{3 s^{2}}\left[\frac{u t-m_{i}^{2} m_{j}^{2}}{s^{2}}\right]\left[-\frac{e_{q} e_{l}\left(L_{l}+R_{l}\right)\left(L_{q}-R_{q}\right)}{8 x_{W}\left(1-x_{W}\right)\left(1-m_{Z}^{2} / s\right)}-\frac{\left(L_{l}^{2}+R_{l}^{2}\right)\left(L_{q}-R_{q}\right)\left(L_{q}+R_{q}\right)}{64 x_{W}^{2}\left(1-x_{W}\right)^{2}\left(1-m_{Z}^{2} / s\right)^{2}}\right],
$$

$L_{l}=S_{j 1} S_{i 1}^{*}\left(2 T_{l}^{3}-2 e_{l} x_{W}\right)$, and $R_{l}=S_{j 2} S_{i 2}^{*}\left(-2 e_{l} x_{W}\right)$ is indeed very sensitive to the slepton mixing matrix $S$ and thus the SUSY parameters, in particular since the squared photon contribution is eliminated. It is also easier implemented experimentally, e.g. at the Tevatron, since protons are much more easily polarized than antiprotons. Neutralinos and charginos also receive $t$ - and $u$ channel contributions from squarks, which couple mostly to their gaugino components and render the analysis slightly more complex [2]. However, the single-spin asymmetry remains also in this case the most interesting observable to determine the gaugino/higgsino mixing and, e.g., the $W$-ino mass parameter $M_{2}$.

\section{Experimental prospects}

Polarized protons can be created from atomic or optically pumped ion sources with a polarization degree $P$ of up to $87 \%$. During acceleration, this polarization is partially lost in resonance crossings, but this can be avoided by introducing Siberian snakes into the storage ring lattice. In 2009 , the RHIC accelerator at BNL has completed a successful $p p$ run with $\sqrt{S}=500 \mathrm{GeV}$ and $P \simeq 50 \%$ and accumulated an integrated luminosity $L$ of about $50 \mathrm{pb}^{-1}$, culminating in the first observation of weak bosons in polarized $p p$ collisions [3]. Several more ten-week polarized $p p$ runs are planned, aiming for $P=65-70 \%$ and $L=266 \mathrm{pb}^{-1}$ in each of the runs [4]. The Tevatron at FNAL is currently operating as an unpolarized $p \bar{p}$ collider at $\sqrt{S}=1.96 \mathrm{TeV}$ and has already accumulated more than $4 \mathrm{fb}^{-1}$ in luminosity. A study of proton beam polarization, performed in the mid-1990s, proposes the replacement of some of the dipole magnets to create room for six Siberian snakes, which would lead again to $P=65-70 \%$ [5]. The LHC at CERN will be operating as an unpolarized $p p$ collider at $\sqrt{S}=7 \mathrm{TeV}$ for two years to reach $L=1 \mathrm{fb}^{-1}$, then at $\sqrt{S}=14 \mathrm{TeV}$ to reach $L=100 \mathrm{fb}^{-1}$. Further upgrades, focusing on even higher luminosity, are currently under discussion. It is interesting to remember that the SSC design had reserved space for Siberian snakes. At the LHC, the required locations would have to be liberated by some of the dipoles.

In Fig. 1 (left) we show the unpolarized hadronic cross sections for pair production of nonmixing tau sleptons at the RHIC, Tevatron, and LHC colliders as a function of their physical mass. Unfortunately, the observation of tau sleptons, as that of any SUSY particles, will be difficult at RHIC, which is the only existing polarized hadron collider. In contrast, tau sleptons will be detectable at the LHC over a large region of the viable SUSY parameter space up to stau masses of about $400 \mathrm{GeV}$. At the Tevatron, the discovery reach extends considerably beyond the current exclusion limits. For a GMSB model with a light tau slepton, we show the single-spin asymmetry in Fig. 1 (right) as a function of the cosine of the stau mixing angle. The asymmetry is quite large and depends strongly on the stau mixing angle. However, very large values of $\cos \theta_{\tilde{\tau}}$ and stau masses below $52 \mathrm{GeV}$ may already be excluded by LEP [6], while small values of $\cos \theta_{\tilde{\tau}}$ may 

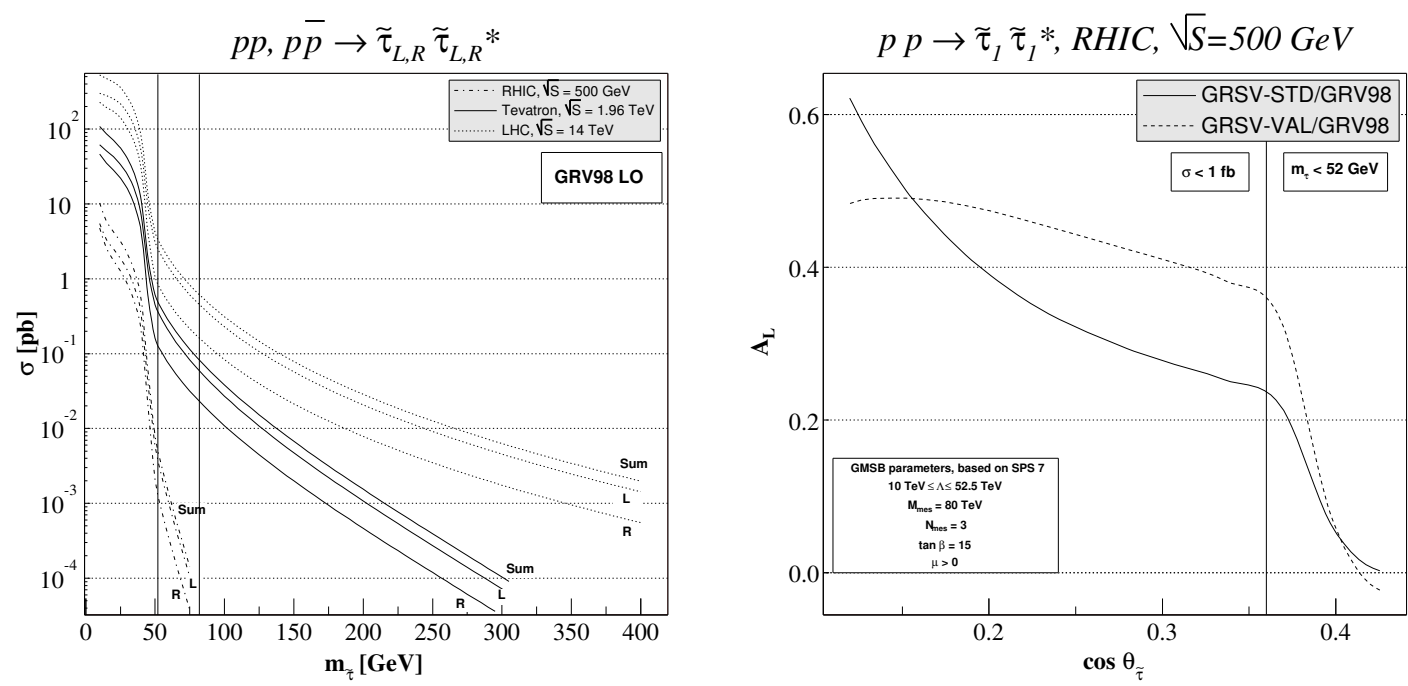

Figure 1: Left: Unpolarized hadronic cross sections for pair production of non-mixing tau sleptons at the RHIC, Tevatron, and LHC colliders as a function of their physical mass. For consistency with the polarized cross sections (see below), GRV98 LO parton densities have been used. The vertical lines indicate two different stau mass limits of 52 and $81.9 \mathrm{GeV}$ [6]. Right: Dependence of the longitudinal single-spin asymmetry $A_{L}$ on the cosine of the stau mixing angle for $\tilde{\tau}_{1}$ pair production in a GMSB model at RHIC.
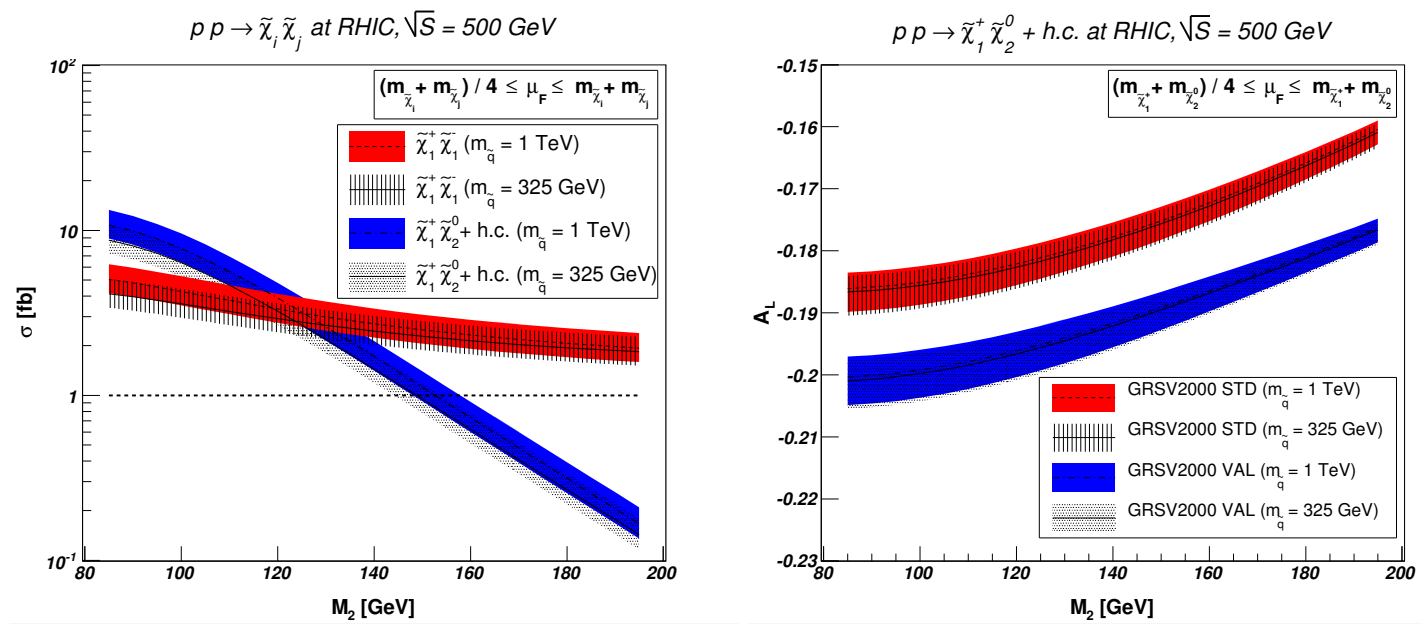

Figure 2: Unpolarized gaugino-pair production cross sections (left) and single-spin asymmetries for chargino-neutralino associated production (right) with $m_{\tilde{\chi}_{2}^{0}} \simeq m_{\tilde{\chi}_{1}^{ \pm}}=80 \mathrm{GeV}$ in $p p$ collisions at RHIC and $\sqrt{S}=500 \mathrm{GeV}$.

be unaccessible at RHIC due to its limited luminosity, which is not expected to exceed $1 \mathrm{fb}^{-1}$. Polarization of the proton beam will also not be perfect, and the calculated asymmetries should be multiplied by the degree of beam polarization $P \simeq 0.7$. The uncertainty introduced by the polarized parton densities increases considerably to the left of the plot, where the stau mass $41 \mathrm{GeV} \leq m_{\tilde{\tau}} \leq$ $156 \mathrm{GeV}$ and the associated values of the parton momentum fractions become large.

In the left part of Fig. 2, we show the total unpolarized cross section for the pair production of the lightest chargino of mass $80 \mathrm{GeV}$ (short-dashed line) and the one for its associated production 
with the second-lightest neutralino (dot-dashed line) at the $p p$ collider RHIC. Both cross sections exceed $1 \mathrm{fb}$ in most of the $M_{2}$ range shown and depend little on the squark mass, indicating that $s$-channel gauge-boson exchanges dominate. We vary the unphysical factorization scale $\mu_{F}$ in the traditional way by a factor of two around the average final state mass (shaded bands; for more precise predictions see $[7,8]$ ). Among the bosons exchanged in the $s$-channel, the $W$-boson is most sensitive to the polarization of the initial quarks and antiquarks, and consequently the single-spin asymmetry for the associated channel, shown in the right part of Fig. 2, reaches large values of around $-20 \%$. As the mass of the neutralino increases and the gaugino fractions of the chargino and neutralino fall up to $M_{2} \leq 200 \mathrm{GeV}$, the cross section and the absolute value of the asymmetry decrease, too. The uncertainty in the scale variation is with $0.5 \%$ considerably smaller than the variation in the asymmetry of $2 \%$, while the uncertainty coming from the polarized parton densities is with $1.5 \%$ of almost comparable size. Single-spin asymmetry measurements for associated chargino-neutralino production at the only existing polarized hadron collider RHIC could therefore be used to determine the gaugino and higgsino components of charginos and neutralinos, provided the polarized quark and antiquark densities are slightly better constrained. The single- and doublespin asymmetries for neutralino pairs reach similar sizes as those for the associated channel, since the left- and right-handed couplings of the $Z$-boson exchanged in the $s$-channel are also different. However, we do not show them here, since the corresponding cross section is unfortunately too small at RHIC. The variation of the asymmetries would, indeed, be quite dramatic: $A_{L}$ changes its sign from $-20 \%$ to $+20 \%$ for $M_{2} \leq 200 \mathrm{GeV}$, and $A_{L L}$ falls from $-5 \%$ to $-20 \%$.

\section{Acknowledgments}

It is a pleasure to thank G. Bozzi, J. Debove and B. Fuks for their collaboration and the organizers of the DIS 2010 conference for creating a stimulating atmosphere despite adverse forces majeures.

\section{References}

[1] G. Weiglein et al. [LHC/LC Study Group], Phys. Rept. 426 (2006) 47.

[2] G. Bozzi, B. Fuks and M. Klasen, Phys. Lett. B 609 (2005) 339; G. Bozzi, B. Fuks and M. Klasen, Phys. Rev. D 72 (2005) 035016; J. Debove, B. Fuks and M. Klasen, Phys. Rev. D 78 (2008) 074020.

[3] B. Surrow for the STAR Collaboration, arXiv:1004.4884.

[4] G. Bunce et al. [RHIC Spin Group], 4-Year Research Plan (2007), http://www.phy.bnl.gov/rhicspin/documents/4year.pdf.

[5] R. Baiod, P. S. Martin and A. D. Russell [SPIN Collaboration], "Polarized Protons in the Tevatron Collider," contribution to the 10th Topical Workshop on Proton-Antiproton Collider Physics, Batavia, Illinois (1995).

[6] R. Barate et al. [ALEPH Collaboration], Phys. Lett. B 433 (1998) 176; J. Abdallah et al. [DELPHI Collaboration], Eur. Phys. J. C 31 (2004) 421.

[7] G. Bozzi, B. Fuks and M. Klasen, Phys. Rev. D 74 (2006) 015001; G. Bozzi, B. Fuks and M. Klasen, Nucl. Phys. B 777 (2007) 157; G. Bozzi, B. Fuks and M. Klasen, Nucl. Phys. B 794 (2008) 46.

[8] J. Debove, B. Fuks and M. Klasen, Phys. Lett. B 688 (2010) 208; J. Debove, B. Fuks and M. Klasen, arXiv:1005.2909. 